\title{
EXUDATIVE DIABETIC RETINOPATHY* SPONTANEOUS CHANGES AND EFFECTS OF A CORN OIL DIET BY
}

\author{
R. C. KING, J. H. DOBREE, D'A. KOK, W. S. FOULDS, AND \\ W. G. DANGERFIELD
}

St. Bartholomew's and North Middlesex Hospitals, London, and Addenbrooke's Hospital, Cambridge

\section{Part I. Spontaneous Changes}

The exudative lesions in diabetic retinopathy have received less attention than the more dramatic changes in the capillaries and veins which make up the characteristic fundus picture. Apart from the occasional fleck found in early cases of diabetic retinopathy the exudates appear in three main forms: a cluster of small deposits, a ring, and a large waxy-looking plaque.

Serial fundus photographs taken at frequent intervals for 2 to 4 years have confirmed the observations of Whittington (1951), Larsen (1960), and other workers, that diabetic exudates disappear spontaneously. There are, however, differences in the evolution and devolution of the three types.

(a) Cluster Form.-This is a collection of small white exudates giving a speckled appearance to a localized area of retina usually a half to one disc diameter in extent. It is often repeated in several parts of the posterior pole and is not generally associated with the normal vessels, aneurysms, or haemorrhages. This cluster form is the most transient type of exudative lesion, and we have observed it to appear and disappear in as short a period as 4 months.

(b) Ring Form (Circinate Retinopathy).-This is of particular interest and has been well described by Houston and Wise (1957). The rings, often incomplete, may appear in two forms: a small lesion from half to one disc diameter across (Fig. 1a) and a larger wreath-like structure some two to three disc diameters across (Fig. 1b), which often encloses the macula. These appearances are not,
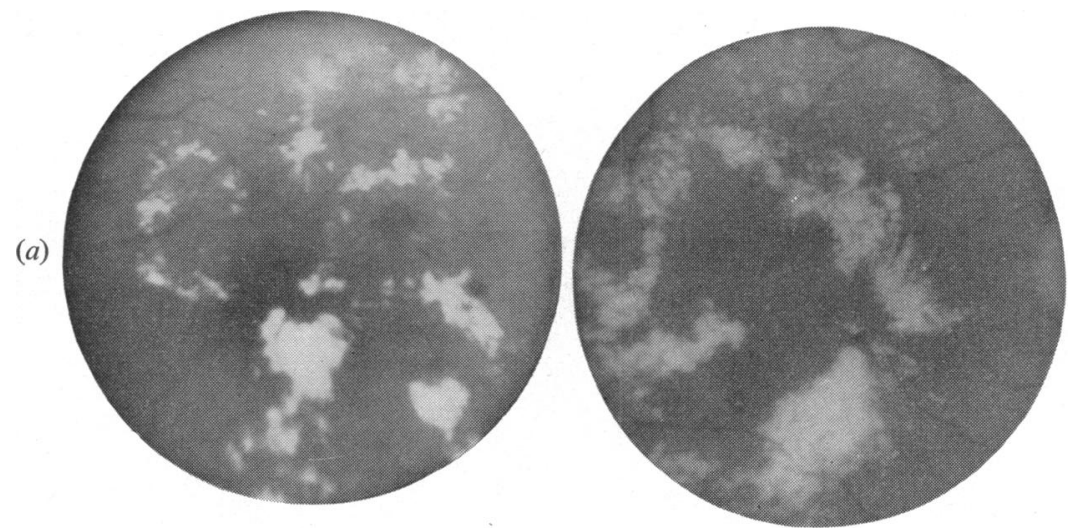

Fig. 1.-Circinate retinal lesions. (a) Small. (b) Large.

* Paper given at a joint meeting of the Irish Ophthalmological Society and the British Diabetic Association held in Dublin, April 25 to 27, 1963. 
however, peculiar to diabetics and are sometimes found in cases of arterio-sclerotic and hypertensive retinopathy.

These circinate lesions not infrequently enclose areas of vascular disturbance such as a small collection of aneurysms or varicosed capillaries, and in these cases appear to be intimately associated with them. Some very clearly demarcated ring lesions may appear to form quite independently from any obvious microscopic capillary lesion, but examination of the serial slides has shown that such exudates have at times appeared in "pale areas" identical in size with the ring which later follows. This appearance, which is of short duration, may represent an area of retinal ischaemia. This change is a slight one and is not always obvious on ophthalmoscopic examination. It is best seen with a light green filter.

The ring forms are relatively stable and have a cycle of appearance and disappearance of 2 to 3 years or longer.

(c) Large Waxy Plaques (see Figs $3 b, 4 a, 6 a, 7 a$ ).-These deposits are often found in the macular region. Examination with the Hruby lens shows them to extend through all the layers of the retina, and the retina itself is often distended to two to three times its normal thickness by what appears to be oedema fluid.

The plaques, the edges of which are ragged, are surrounded by fluid and have an appearance not unlike sugar or some other crystalline substance forming or dissolving in water. Of the three exudate forms these waxy plaques are the most stable and probably remain in the same sites for years, eventually undergoing further degenerative changes.

An important point in the evolution of the exudates is that, although the general rule is the gradual appearance and disappearance of the lesions (some groups progressing at the same time as others are regressing), we have never found any evidence of migration of lipoid from one site to another. This may be due either to the gradual absorption of ultra-microscopic particles or to the fact that phagocytosed fat is invisible to ordinary fundus lighting (Wolter, Goldsmith, and Phillips, 1957). Wolter (1961) and Toussaint, Cogan, and Kuwabara (1962) have demonstrated histologically that the lipoid material is eventually phagocytosed and possibly removed via the local blood vessels.

Transient soft exudates, very similar to those found in hypertensive retinopathy, were frequently seen; they lasted only a few weeks and left no trace of their presence.

\section{Field Changes associated with Exudative Lesions}

In general, as will be described later, the disappearance of the central exudates was not accompanied by the corresponding increase of visual acuity that had been hoped for. Bjerrum screen examination of the $7^{\circ}$ or so of retina surrounding the macula showed that scotomata had developed in areas of the retina which had previously been involved in patches of exudate but which were now clear. Poor central fixation may make this type of central field examination unreliable.

A more accurate method of localizing the exact spot on the retina which is being stimulated is to project a shadow test object in the ophthalmoscope 
beam and thus to stimulate and observe the point of stimulus at one and the same time. The Visuscope, which is used in determining the fixation area in cases of strabismus, is especially useful in this connexion. It was found that the black star was invisible to the patient when it was projected on to an exudate or, more important, on to an area which had previously contained one.

These observations support the view (Wolter, 1961; Bloodworth, 1962) that diabetic exudates are the result of degeneration of the retinal neurons and that these islets of damage, although rendered invisible by subsequent absorption, have a permanent effect on vision.

\section{Part II. EfFect OF a Corn OIL Diet}

The fatty material responsible for the exudates is partly intra- and partly extra-cellular. Its origin and the relationship between the intra- and extracellular components are still the subject of controversy; some believe its deposition to be due to a combination of increased plasma lipid levels and increased capillary permeability and others to neuronal degeneration.

Despite the controversy as to which comes first-the neuronal degeneration or the exudate-it seemed to us that anything that could reduce the extent of the exudate might be followed by an improvement in vision. Kempner (1945) had noted marked improvement in exudative lesions in two diabetics given the rice diet for their hypertension and van Eck (1959) described considerable reduction in extent of exudate in five of ten patients whose animal fat intake had been restricted to $20 \mathrm{~g}$. daily.

There is considerable evidence to suggest that a diet containing appreciable quantities of unsaturated fatty acids leads to a reduction in serum lipid content, and it was felt that such a diet coupled with a drastic reduction of animal fat might assist in the treatment of exudative diabetic retinopathy, particularly as diabetics tend to have raised serum lipid levels.

For the last 3 years we have observed a total of forty patients with exudative retinopathy by means of serial retinal photographs: 23 patients (37 eyes) continued with their normal animal fat consumption while seventeen (26 eyes) reduced their daily intake to $20 \mathrm{~g}$. with the addition of $60 \mathrm{~g}$. of unsaturated vegetable fat per day, the latter being provided by Alfonal Products. The two groups did not differ materially with regard to age, sex, duration of diabetes or treatment, and the corn oil diet did not present any major practical problems. The requisite amount of unsaturated fatty acid can be provided in many ways of which the following is an example:

Alfonal corn oil $1 \mathrm{oz}$.

Alfonal margarine $1 \frac{1}{2}$ ozs.

Alfonal cheese spread 2 portions

Alfonal biscuit 1

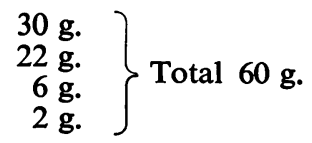

Variations in serum lipid levels were studied in some patients in the earlier stages of the trial; both total lipid and serum cholesterol levels fell in most of them with some fluctuation (Fig. 2, opposite). 
○- Total serum lipid

— $750 \mathrm{mg} . / 100 \mathrm{ml}$. Upper limit of normal

Serum Cholesterol

$250 \mathrm{mg} . / 100 \mathrm{ml}$. Upper limit of normal
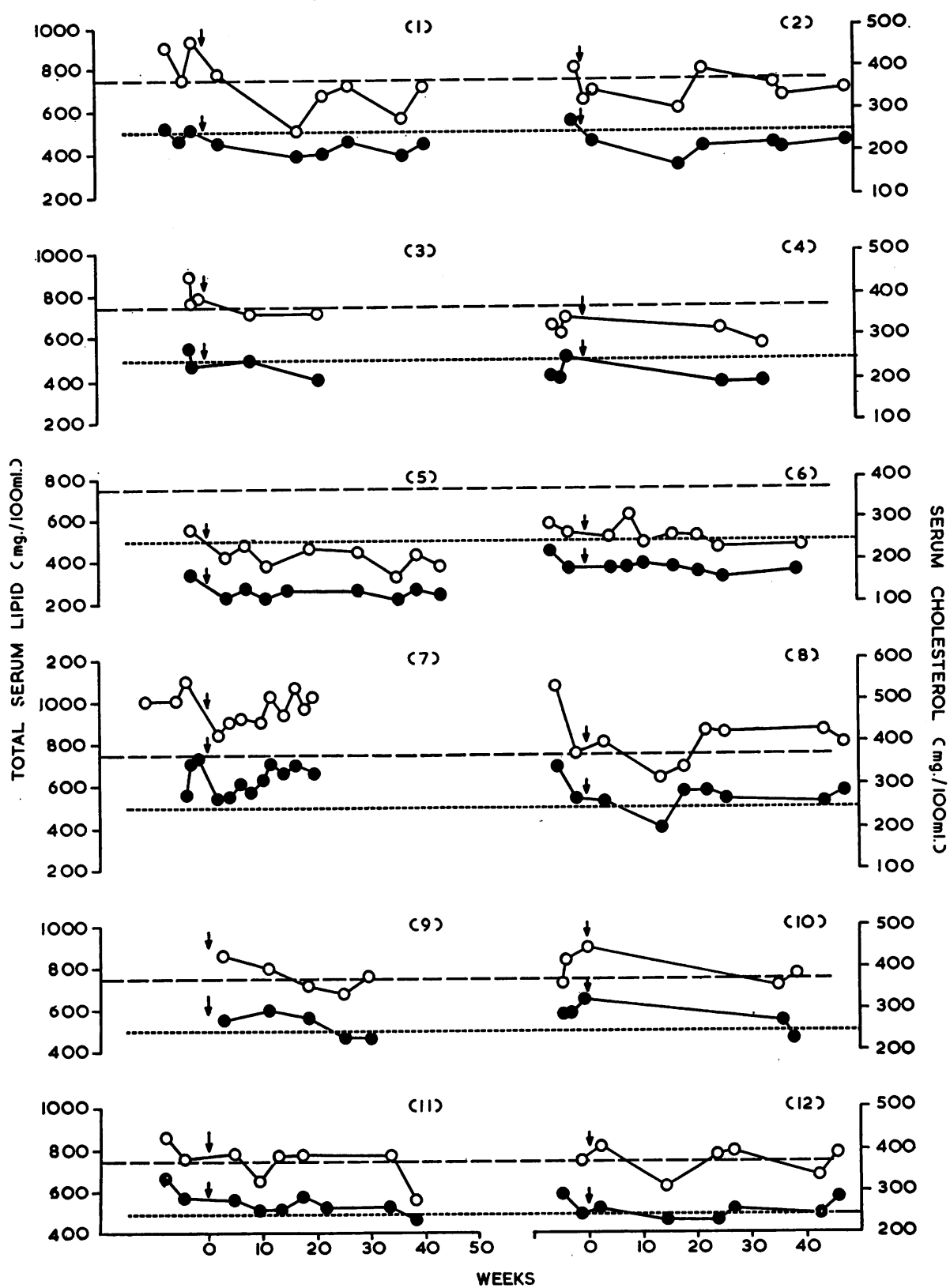

Fig. 2.-Variations in total serum lipid and serum cholesterol levels in twelve patients on a corn oil diet. 
The effect of the regime on diabetic control, insulin requirements, and weight was not significant.

The most striking change in the treated group was a marked reduction in the extent of the retinal exudate.

Of the 26 treated eyes, $21(80.8$ per cent.) were improved, some dramatically so, and two ( 7.7 per cent.) became worse; of the 37 untreated eyes, eleven ( 29.7 per cent.) were improved and 22 (59.5 per cent.) became worse. Changes in microaneurysms were not studied, chiefly because of the difficulty of identifying them on retinal photographs, and we were not concerned with patients with proliferative lesions. Variations in extent of haemorrhage were noted; in the treated group 50 per cent. were improved and 20 per cent. worse, the remainder being unchanged, and in the untreated group 27 per cent. were better and 20 per cent. worse. Examples are shown in Figs 3 to 7 .

(a)

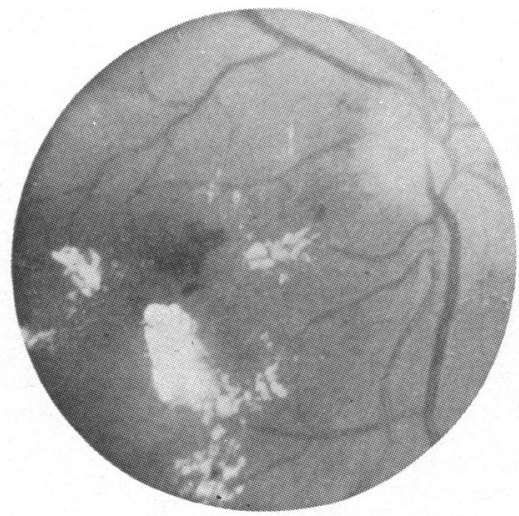

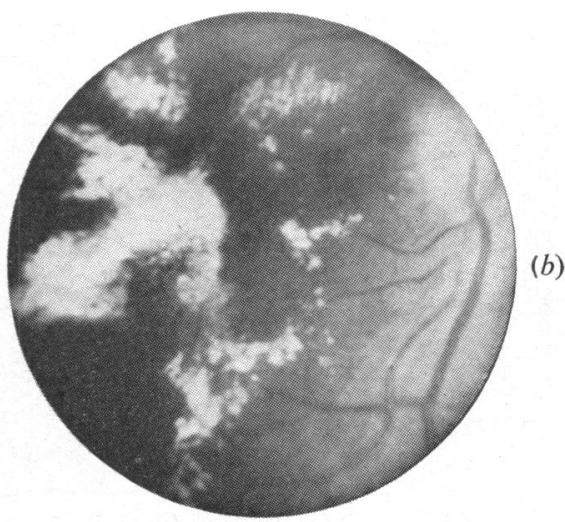

Fig. 3.-Right eye of untreated case.

(a) Initial appearance, (b) 18 months later.

(a)

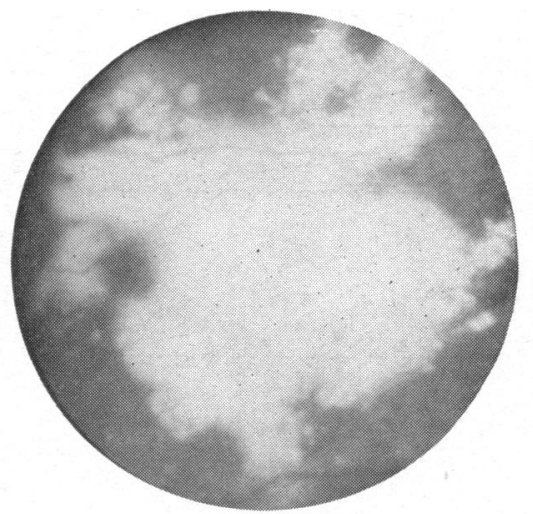

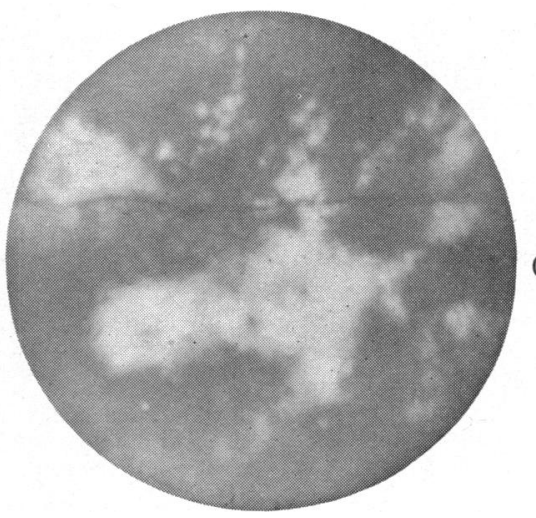

(b)

FIG. 4.-Treated case. (a) Before. (b) One year after treatment. 
(a)

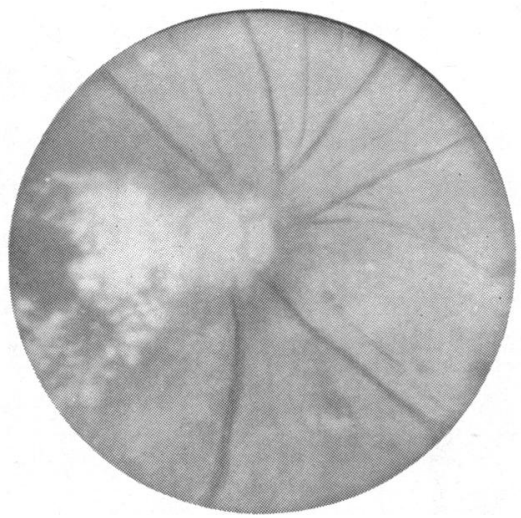

FIG. 5.-Right eye of treated case.

(a)

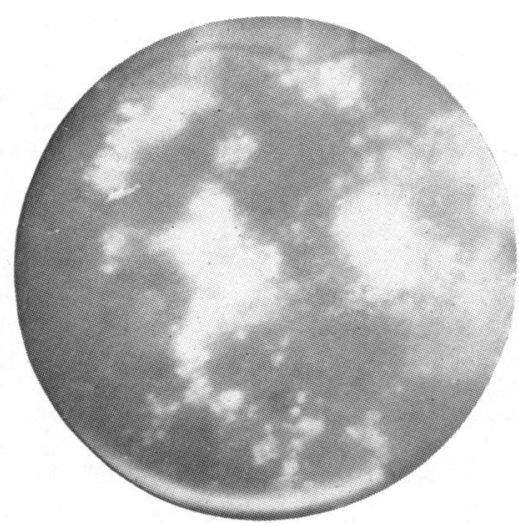

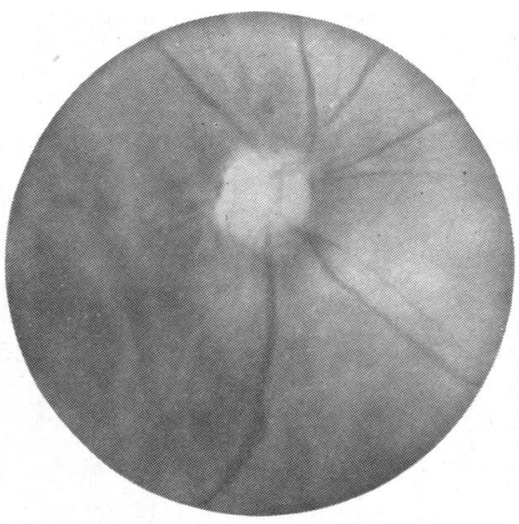

(b)

(a) Before. (b) 2 years after treatment.

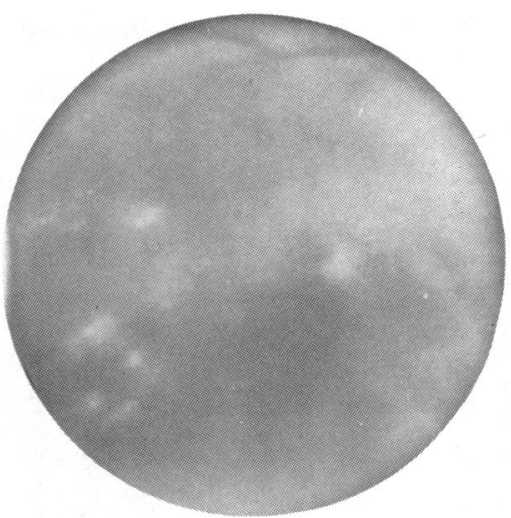

(b)
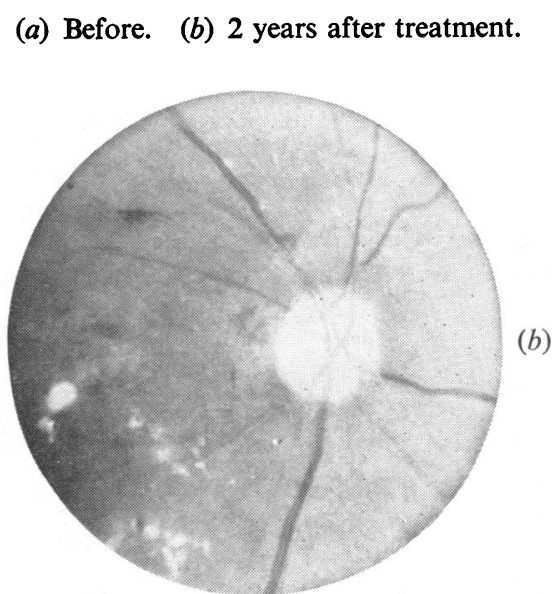

(b) 3 years after treatment. 
It was disappointing to find no striking improvement in visual acuity despite the very marked reduction in the extent of the exudate. In both treated and untreated groups some patients showed a tendency towards slight improvement, probably because of increasing familiarity with the procedure, but there were no significant differences.

\section{Summary}

PART I. The form of diabetic exudates, their evolution and devolution, and the field defects associated therewith are described.

PART II. The results suggest that, by means of a diet containing $20 \mathrm{~g}$. animal fat and $60 \mathrm{~g}$. unsaturated fat, it is possible to achieve a significant reduction in the amount of exudate present in diabetic retinopathy. There is, however, no associated improvement in visual acuity, and it therefore seems likely that the exudate is the end-result of neuronal degeneration which itself impairs vision.

We are grateful to Dr. K. O. Black for permission to study his patients, and to Alfonal Limited for free supplies of corn oil products.

\section{REFERENCES}

BLoodworth, J. M. B. (1962). Diabetes, $2,1$.

Houston, W. R., and WISE, G. N. (1957). A.M.A. Arch. Ophthal., 58, 777.

KEMPNER, W. (1945). N.C. med.J., 6, 117.

LARSEN, H. W. (1960). Acta ophthal. (Kbh.), Suppl. 60

Toussaint, D., Cogan, D. G., and Kuwabara, T. (1962). Arch. Ophthal., 67, 42.

VAN ECK, W. F. (1959). Amer.J. Med., 27, 196.

WhitTIngton, T. H. (1951). Proc. roy. Soc. Med., 44, 753.

WOLTER, J. R. (1961). Amer. J. Ophthal., 5i, 1123.

—, Goldsmith, R. I., and Phillips, R. L. (1957). A.M.A. Arch. Ophthal., 57, 376. 